\title{
Glycemic control in a 79-year-old female with mild cognitive impairment using a medication reminder device: a case report
}

\author{
T. Kamimura ${ }^{1}$ and $\mathrm{H}$. Ito ${ }^{2}$ \\ ${ }^{1}$ School of Health Sciences, Shinshu University, 3-1-1 Asahi, Matsumoto, Nagano Pref. 390-8621, Japan \\ ${ }^{2}$ Department of Social Psychiatry, National Institute of Mental Health, National Center of Neurology and Psychiatry, 4-1-1 Ogawa-Higashi, Kodaira, \\ Tokyo 187-8553, fapan
}

ABSTRACT

A 79-year-old female with type 2 diabetes and mild cognitive impairment (Clinical Dementia Rating score of 0.5 ) was supported with medication with regard to the daily requirements using a medication reminder device. Use of this device not only improved her medication adherence, hemoglobin A1c level, and selfconfidence but also reduced caregiver's burden. For elderly patients with such diseases, loading the device with medication, providing advance notice before mechanical reminders for a short period after the device's activation, monitoring unused medication, and adjusting the timing of reminders according to users' daily routine, seemed to facilitate daily use of the device.

Key words: mild cognitive impairment $(\mathrm{MCl})$, diabetes, memory

\section{Introduction}

Adherence to long-term therapies remains a major problem in medical care (WHO, 2003). Medication adherence in patients with type 2 diabetes, which is a major chronic disease, was estimated as $61-85 \%$ (Cramer, 2004).

Cognitive impairment presents further challenges to compliance with a medication regimen (Campbell et al., 2012). Simple reminders such as a medication reminder device (Holthe, 2004; Sather et al., 2007; Kamimura et al., 2012) or automated phone calls (Ownby et al., 2012) have been reported to improve medication adherence among elderly patients with cognitive impairment. Although human support could be essential for the use of this device, this issue has not been addressed. In addition, little attention has been paid on the pharmacological and psychosocial effects of such devices. This may be a reason for such devices to have not been widely used in patients with cognitive impairment.

This case report provides insight into the type of human support that enables elderly patients with both cognitive impairments and chronic diseases, such as type 2 diabetes, to establish the daily use

Correspondence should be addressed to: T. Kamimura, School of Health Sciences, Shinshu University, 3-1-1 Asahi, Matsumoto, Nagano Pref. 3908621, Japan. Phone/Fax: +81-263372395. Email: tkamimu@shinshu-u.ac.jp. Received 1 Oct 2013; revision requested 28 Oct 2013; revised version received 14 Nov 2013; accepted 15 Nov 2013. First published online 2 January 2014. of such a device through observation of the device's pharmacological and psychosocial effects.

\section{Case presentation}

This report focused on a 79-year-old female diagnosed with type 2 diabetes and mild cognitive impairment (MCI), with a Clinical Dementia Rating score of 0.5 and Mini-Mental State Examination score of 30 . She lived independently with her husband who had been diagnosed with Alzheimer's disease. Her daughter often visited home to help them. She was prescribed oral hypoglycemic agents along with anti-dementia medication. Oral hypoglycemic agents (Glinides and Biguanide) were prescribed for three times a day before each meal, while anti-dementia agents were prescribed for once a day after breakfast. Her daughter often reminded her to take her medication either in person or by phone. However, she sometimes still forgot the medication, which led to inadequate glycemic control (hemoglobin A1c (HbAlc), 8.0\%).

We, therefore, assessed the effects of the medication reminder device (Pivotell Ltd, UK; Figure 1). This device sounds an alarm and displays a flashing light to remind the user when to take his/her medication. When the alarm rings, the correct medication dose is automatically dispensed into an opening in the lid. The user must 
Table 1. Requirements for using the medication reminder device

Adequate auditory, visual, and finger dexterity required to manipulate the device.

Able to comprehend that the alarm is a reminder to take medication.

Able to return the device to the correct position after retrieving the medication. ${ }^{*}$

Habit of taking medication regularly at home, albeit with occasional lapses and with assistance from someone else.

Medication in the form of tablets or capsules.

Caregivers available to load the device with medication and check the amount of unused medication.

Note: * If the device is in an inverted position at the alarm time, the warning beep sounds, and this ability is included in these requirements.
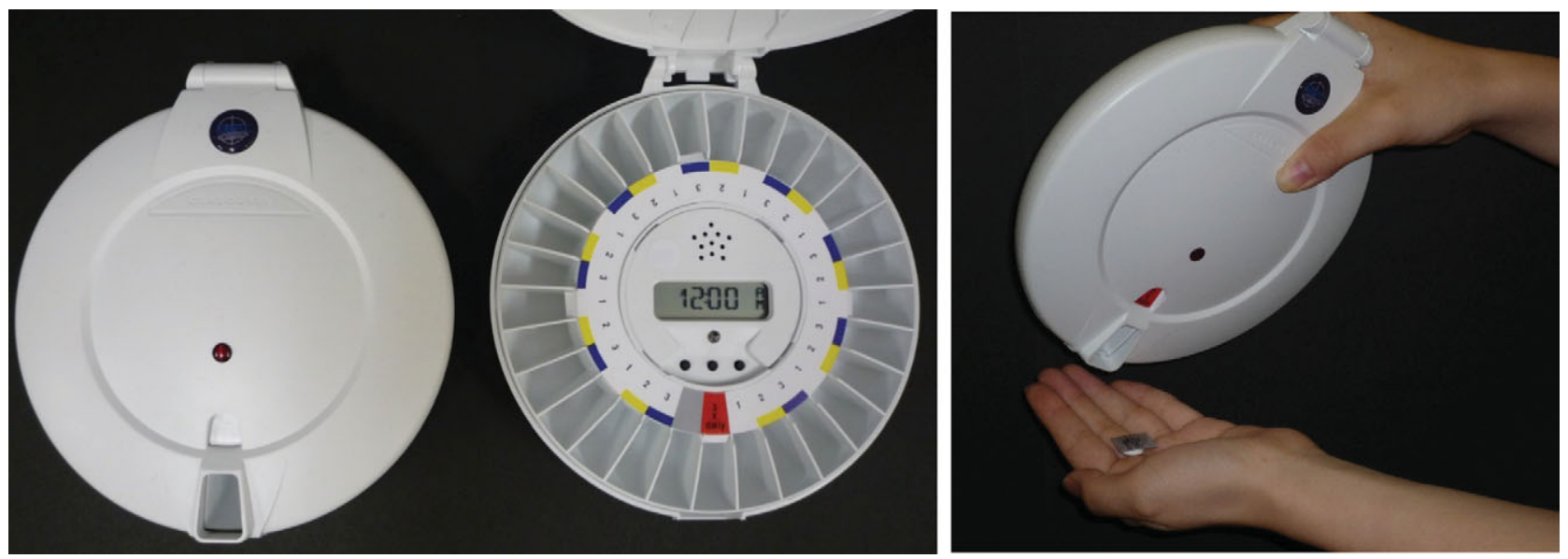

Figure 1. (Colour online) The medication reminder device (Pivotell Ltd, UK).

then invert the device to retrieve the medication and stop the alarm. The patient has to fulfill the following requirements for using the device: adequate auditory, visual, and finger dexterity ability; ability to comprehend that the alarm is a reminder to take medication; and the habit of taking medication regularly at home, albeit with occasional lapses and with other person's assistance (Table 1).

The device was initially used to provide doses twice a day (before lunch and dinner). To limit the burden of learning to use this new device, its use was limited to only part of her prescription. Because of her routine outings once a week, help was provided only at the time of medication. However, no help was provided for morning doses.

Her daughter assisted in loading the device with medication, providing advance notice in person or by phone before automatic reminders, monitoring unused medication, and adjusting the timing of reminders according to her mother's daily routine.

According to the amount of unused medication as determined by her daughter, medication adherence improved from $64 \%$ (five missed doses per week) in one week before activation of the device to $86 \%$ (two missed doses per week) one week later. The reason for these two missed doses after activation was a non-routine outing for dinner.

After observing these positive results, the number of instances for the daily administration of medication using the device was increased from two to three times a day (before each meal). In addition, when family members accompanied her during outings, they reminded her to take the medication. Approximately two weeks after using this device, she needed assistance from her daughter only to load the device with medication and to check for unused medication.

After a month, medication adherence improved to $95 \%$ (one missed dose per week), and HbA1c decreased to $7.8 \%$. She reported an increase in self-confidence because of these improvements. According to a four-point ordinal scale, her daughter's burden while assisting to maintain the medication regimen decreased from " 3 : mild burden" before activation of the device to " 2 : little burden" after one month of usage. Both patient and her daughter reported the device as helpful, especially in relieving their worries about taking medication. After three months, medication adherence was $100 \%$, and $\mathrm{HbA} 1 \mathrm{c}$ was $6.9 \%$. Her daughter reported " 1 : no burden" on ordinal scale. After six months, medication adherence was $95 \%$ (one missed dose per week), and $\mathrm{HbAlc}$ was $7.1 \%$. Her daughter reported " 2 : little burden" with regard to the level of assistance necessary in maintaining her mother's medication regimen. After 15 months the patient continued to use the device. Her daughter said: "Though the cognitive impairment of my mother have deteriorated, she can continue to use the device. It is very helpful for my 
family because we need not worry about her taking medication in spite of the increasing difficulties of my mother's daily life."

\section{Discussion}

Our findings suggest that an individual with MCI and without any other mental/physical disability and who has acquired the habit of taking medication regularly at home, albeit with occasional lapses and with help from others, is a suitable candidate to use a simple reminder device. In addition, loading the device with medication, providing advance notice before automatic reminders for a short period after the device's activation, monitoring unused medication, and adjusting the timing of reminders according to user's daily routine, seemed to facilitate its daily use.

Sohlberg and Mateer (2001) stated that an effective strategy for facilitating the use of external aids to assist adults and children with cognitive impairments included systematic prompting, performance monitoring, and errorless learning. Our findings, which followed Sohlberg and Mateer's (2001) recommendations, revealed that the use of such methods could be beneficial in improving adherence to medication regimens in elderly patients.

Similar to previous studies (McCurry et al., 2010; Ownby et al., 2012), our report showed that a caregiver is likely to be a determinant in adherence to therapy in elderly patients with cognitive impairments. The assistance provided by the caregiver in this study, however, may not be typical, and therefore a focus on reducing the caregiver's burden may promote the use of such a device. Other strategies may include alternative prescriptions with a decreased daily dose frequency.

This study showed that elderly patients with type 2 diabetes and cognitive impairments may benefit from the medication reminder device. The American Diabetes Association recommended an HbA1c level of $<7.0 \%$ for most patients and a slightly higher level $(7.5-8.0 \%)$ for frail elderly patients (Inzucchi, et al., 2012). The device was effective in improving glycemic control according to these clinical criteria.

Furthermore, with regard to the psychosocial effects, our observations suggest that the medication reminder device, in addition to caregiver's assistance, may increase self-confidence in elderly people with cognitive impairments and decrease caregivers' burden.

One limitation of this technique is that it cannot prevent patients from forgetting to take the medicine after retrieving it from the device, and this can easily occur in elderly patients with cognitive impairments. To compensate this limitation, knowledge of the patient's previous medication experiences and adjustment with the timing of the reminders according to his/her daily routines are necessary. Further modifications are required for a patient without such habits.

Further studies are required to validate the medication reminder device's pharmacological and psychosocial effects, which we found in this study, because these results were based on a case report. Furthermore, to clarify a list of qualifiers for using such a device among elderly patients with cognitive impairments, an additional evaluation study is required on the suggested requirements as determined in our study (Table 1).

\section{Conflict of interest}

None.

\section{Description of authors' roles}

T. Kamimura formulated the study question and wrote the paper. H. Ito assisted in writing the paper.

\section{Acknowledgments}

The patient provided written informed consent to publish this paper.

\section{References}

Campbell, N. L., Boustani, M. A., Skopelua, E. N., Gao, S., Unverzagt, F. W. and Murray, M. D. (2012). Medication adherence in older adults with cognitive impairment: a systematic evidence-based review. American Fournal of Geriatric Pharmacotherapy, 10, 165-177.

Cramer, J. A. (2004). A systematic review of adherence with medications for diabetes. Diabetes Care, 27, 1218-1224.

Holthe, T. (2004). Enabling technologies for people with dementia: national report on results from Norway. Available at: http://www.enableproject.org/download/ Enable\%20-\%20National\%20Report\%20-\% 20Norway.pdf; last accessed 20 September 2013.

Inzucchi, S. E. et al. (2012). Management of hyperglycemia in type 2 diabetes: a patient-centered approach. Diabetes Care, 35, 1364-1379.

Kamimura, T., Ishiwata, R. and Inoue, T. (2012). Medication reminder device for the elderly patients with mild cognitive impairment. American fournal of Alzheimer's Disease and Other Dementias, 27, 238-242.

McCurry, S. M., Pike, M. C., Logsdon, R. G., Vitiello, M. V., Larson, E. B. and Teri, L. (2010). Predictors of short-and long-term adherence to a daily walking program 
in persons with Alzheimer's disease. American fournal of Alzheimer's Disease and Other Dementias, 25, 505-512.

Ownby, R. L., Hertzog, C. and Czaja, S. J. (2012).

Tailored information and automated reminding to improve medication adherence in Spanish- and English-speaking elders treated for memory impairment. Clinical Gerontologist, 35, 221-238.

Sather, B. C., Forbes, J. J., Starck, D. J. and Rovers, J. P. (2007). Effect of a personal automated dose-dispensing system on adherence: a case series. Fournal of the American Pharmacists Association, 47, 82-85.

Sohlberg, M. M. and Mateer, C. A. (2001). Cognitive Rehabilitation: An Integrative Neuropsychological Approach. New York, NY: Guilford Press.

WHO. (2003). Adherence to Long-Term Therapies: Evidence for Action. Available at: http://www.who.int/chp/knowledge/ publications/adherence_report/en/; last accessed 20 September 2013. 